\title{
Presentación atípica de una tiroiditis supurativa en una paciente inmunocompetente con compromiso vascular (síndrome de Lemierre)
}

\author{
Case report: atypical presentation of suppurative thyroiditis in an \\ immunocompetent patient with vascular involvement (Lemierre's \\ syndrome)
}

Rojas $W^{1}$, Tovar $H^{2}$, Vargas JJ', Tous $R^{3}$.

${ }^{1}$ Endocrinólogo, Jefe, Servicio de Endocrinología, Hospital San José ${ }^{2}$ Endocrinólogo, Docente FUCS, Hospital de San José.

${ }^{3}$ Fellow en Endocrinología, FUCS, Hospital de San José.

Fecha de recepción: 18/07/2019

Fecha de aceptación: 31/07/2019

\section{Resumen}

La patología de la glándula tiroidea, en su mayor porcentaje, está constituida por las tiroiditis, una amplia gama de enfermedades de diversa etiología (infecciosa, autoinmune o de ocurrencia desconocida) y su duración (agudas, subagudas o crónicas). Desde el punto de vista fisiológico pueden variar en su presentación y causar hipotiroidismo o tirotoxicosis o no alterar el funcionamiento de las hormonas tiroideas.

La tiroiditis supurativa aguda tiene una causa principalmente infecciosa. Es una condición inusual, ya que la tiroides normal es particularmente resistente a las infecciones debido a su extenso drenaje venoso y linfático, además de su alto contenido de yodo y la presencia de una cápsula fibrosa, que sirve como escudo ante agentes nocivos externos. Las infecciones de la tiroides representan aproximadamente el 0,7\% de toda la patología quirúrgica en relación con esta glándula ${ }^{(1-3)}$ y son más frecuentes en una tiroides anormal. A pesar de lo extraño de esta condición, es importante conocer cuál es su presentación y su asociación con los pacientes inmunocompetentes, como la extensión vascular presente en el síndrome de Lemierre que presentó esta paciente.

El objetivo de este informe es presentar un caso clínico de una paciente que cursó con un episodio de tiroiditis infecciosa, su manejo y las diferentes causas de tiroiditis. Se realizó una revisión actualizada del tema y las diferentes opciones de tra- tamiento. Este caso se manejó rápidamente con corticoides y terapia antibiótica, al igual que drenaje quirúrgico.

La paciente se recuperó respondiendo satisfactoriamente al manejo quirúrgico y farmacológico instaurado. Se concluyó que el tratamiento efectuado fue exitoso.

Palabras clave: tiroiditis, tiroiditis supurativa.

\section{Abstract}

The pathology of the thyroid gland, in its highest percentage, is constituted by thyroiditis, a wide range of diseases of different etiology (infectious, autoimmune or of unknown occurrence) and duration (acute, subacute or chronic). From the physiological point of view, they can vary in their presentation, causing hypothyroidism or thyrotoxicosis, or not altering the functioning of thyroid hormones.

Acute suppurative thyroiditis has a mainly infectious cause. It is an unusual condition, since the normal thyroid is particularly resistant to infections due to its extensive venous and lymphatic drainage, in addition to its high iodine content and the presence of a fibrous capsule, which serves as a shield against harmful external agents. Thyroid infections represent approximately $0.7 \%$ of all surgical pathologies in relation to this gland (1-3) and are more frequent in an abnormal thyroid gland. Despite the strangeness of this condition, it is important to know what its presentation is and its association in immunocompetent patients such as the vascular extension present in Lemierre's syndrome, which this patient presented.

The objective of this report is to present a clinical case of a patient who had an episode of infectious thyroiditis, its management and the different causes of thyroiditis. An updated review of the topic and the different treatment options was carried out. 
This case was handled quickly with corticosteroids and antibiotics, as well as surgical drainage.

The patient recovered responding satisfactorily to the surgical and pharmacological management established. It is concluded that the treatment carried out was successful.

Keywords: thyroiditis, supperative thyroiditis.

\section{Informe de caso}

Paciente femenino de 24 años de edad sin antecedentes de importancia y ocupación de ama de casa. Consultó inicialmente a un hospital local de tercer nivel por un cuadro clínico de 45 días de evolución de la aparición de una tumefacción en la región cervical, zona IV del cuello, con rápido crecimiento y dolorosa a la palpación. La paciente refiere que inicialmente le realizaron estudios imagenológicos con hallazgo de nódulo tiroideo (se desconocen las características) y estudios bioquímicos que se interpretan inicialmente como hipertiroidismo. Recibió manejo con metimazol 10 mg Vo/día y propanolol 40 $\mathrm{mg}$ VO/día, con posterior egreso. La paciente reingresa por no presentar resolución del cuadro. Además, en los últimos 8 días presentó incremento progresivo del tamaño de la masa (figura 1) y dolor intenso sobre la misma asociado con disfagia, por lo que decide consultar a nuestra institución.

Figura 1. Masa indurada en región lateral izquierda del cuello

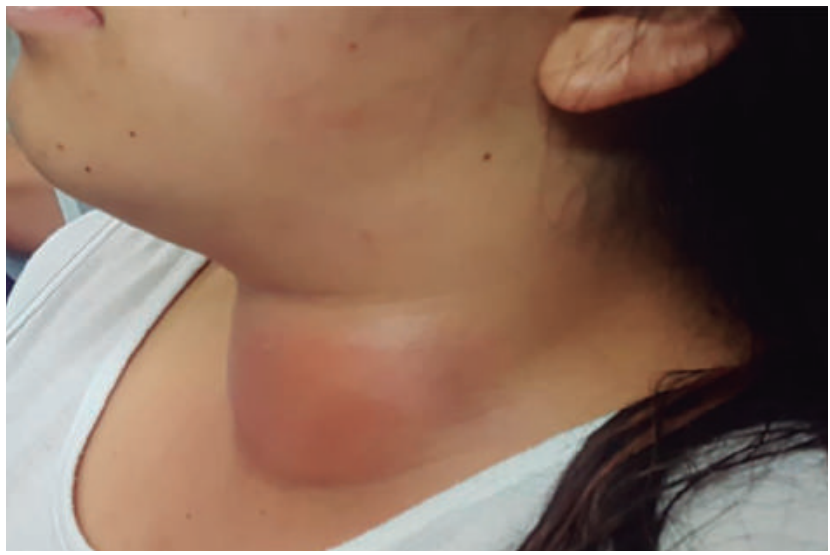

Ingresó al servicio de urgencias y fue valorada inicialmente por el servicio de cirugía de cabeza y cuello, negando fiebre. En el examen se encontraba álgida y el único hallazgo positivo en el examen físico del cuello fue el aumento del diámetro anteroposterior, bocio grado 3 y presencia de masa tumefacta lateralizada a la izquierda, con edema, calor local, rubor y dolor, sin adenomegalias y signo de Pemberton negativo.

La paciente aportó en una hospitalización previa una TSH menor de 0,015 ng/mL. No aportó ecografía ni T4 libre. Al ingreso se obtuvo analítica sanguínea que mostró un hemograma con leucocitosis de $25400 \mathrm{~mm}^{3}$ a expensas de neutrófilos
$24200 \mathrm{~mm}^{3}$, hemoglobina de 10,3 gramos y plaquetas normales; lactato sérico elevado y gases arteriales con acidosis metabólica leve, transaminasas ligeramente elevadas (TGO 67, TGP 60) además de T4 libre: sodio, potasio, calcio, BUN, creatinina, fosfatasa alcalina y bilirrubinas normales, además de serología para VIH, la cual fue negativa.

Se realizó una tomografía axial computada (TAC) de cuello contrastado (figura 2) que reportó la presencia de una masa sólida heterogénea con áreas de necrosis en la topografía del lóbulo tiroideo izquierdo, con efecto compresivo sobre la tráquea que la desvía hacia la derecha sin disminución significativa del calibre y presencia de reacción desmoide, hallazgos relacionados, probablemente, con neoplasia primaria de glándula tiroides y adenomegalias de aspecto reactivo bilaterales. Como hallazgo adicional, hubo colapso de la vena yugular interna en el trayecto proximal adyacente a la masa descrita con posterior opacificación (la imagen Doppler confirmó la presencia de trombosis parcial de la vena yugular interna izquierda de características subaguda frente a crónica recanalizada).

Figura 2. Masa sólida, heterogénea, con áreas de necrosis en el lóbulo tiroideo izquierdo, con efecto compresivo sobre la tráquea desviándola hacia la derecha

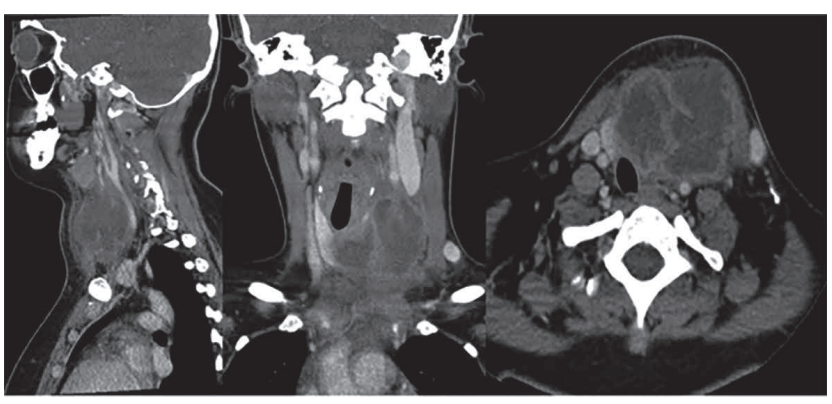

La paciente fue valorada por el servicio de endocrinología, que consideró el cuadro clínico además de los paraclínicos solicitados en el contexto de la presencia de una masa heterogénea en la TAC, con realce periférico y áreas de atenuación entre 90 y 25 unidades Hounsfield (uh). Se consideró como primera opción diagnóstica tiroiditis supurativa aguda frente a neoplasia tiroidea sobreinfectada. Dada la impresión diagnóstica, la paciente no se beneficiará del uso de antitiroideos, pero sí del manejo antibiótico de amplio espectro, por lo que se inició el manejo con ampicilina/sulbactam y se sugirió la toma de hemocultivos y aspiración del contenido de la lesión con toma de muestras para cultivo y biopsia. Para una mejor caracterización de la lesión, se realizó una nueva ecografía del cuello que mostró una masa dependiente de lóbulo tiroideo izquierdo, con áreas centrales sugestivas de colección, confirmando el diagnóstico de tiroiditis supurativa con compromiso vascular adyacente (síndrome de Lemierre). Se llevó a drenaje guiado por ecografía, en la que se encontró una colección de aspecto lateral izquierdo 
de cuello de 63 x 52 x 16 mm y volumen de $28 \mathrm{~mL}$. Se instauró catéter para drenaje y se obtuvieron $20 \mathrm{~mL}$ de material purulento y se enviaron muestras para cultivos, los cuales fueron positivos para Streptococcus anginosus a las 72 horas, sensibles a la penicilina, así como hemocultivos a las 48 horas positivos para cocos grampositivos (Peptostreptococcus $\mathrm{sp}$.).

A los 3 días posteriores desde el inicio del drenaje percutáneo, por parte de radiología se realizó un control ecográfico sin encontrar regresión de la colección, por lo que fue llevada a exploración de cuello más drenaje quirúrgico, obteniendo aproximadamente $100 \mathrm{~mL}$ de material purulento y tejido necrótico. Se envió muestra para cultivo, que confirmó el germen anaerobio procedente de la cavidad oral.

La paciente completó 14 días de antibiótico (ampicilina/ sulbactam), con resolución total de los síntomas. Previo al egreso, se realizó una ecografía de control que mostró una pequeña colección residual a nivel del istmo. La paciente asistió a su control ambulatorio 2 semanas después con una ecografía en la que se informa un parénquima de forma, tamaño y posición de características usuales, sin evidencia de lesiones difusas, que llama la atención por la asimetría e irregularidad del lóbulo izquierdo y presencia de una lesión de 8,9 x 5,0 mm de contornos irregulares mal definidos, sin evidencia de alteración de los vasos del cuello.

\section{Discusión}

La mayor parte de la patología de la glándula tiroides está constituida por las tiroiditis (granulomatosa, de Quervain, linfocítica e inducida por radiación), condiciones autoinmunes (enfermedad de Hashimoto, enfermedad de Graves), neoplasias primarias y metástasis, hemorragia de un quiste preexistente, tirotoxicosis asociada con amiodarona, infarto, amiloidosis y sarcoidosis.

La presentación más frecuente de la tiroiditis supurativa es la aguda, una condición poco prevalente que tiene una presentación variada según la ruta de diseminación, el tipo de microorganismo causal y la inmunocompetencia del huésped. Sin embargo, la inferencia es que una tiroides normal es resistente a la infección por su extenso drenaje venoso y linfático ${ }^{(4)}$, y la infección suele ocurrir cuando los microorganismos alcanzan la tiroides por siembra linfática o hematógena de esta glándula altamente vascularizada ${ }^{(2,5)}$ o logran propagarse desde sitios adyacentes de la infección (esófago, orofaringe, oído medio) a través de la fascia, aunque esto es menos frecuente, ya que la tiroides está encapsulada por una vaina fibrosa ${ }^{(6)}$.

Los microorganismos grampositivos predominan como agentes. Los etiológicos de mayor frecuencia son los Staphylo- coccus (más común aureus), se identificaron como agentes causales de un tercio de los casos con cultivo positivo. El síntoma clínico dominante es el dolor en la región cervical anterior, a nivel de la glándula tiroides, que progresivamente aumenta de tamaño y se asocia con un edema firme de la glándula y calor local, que típicamente se desplaza durante la deglución.

El análisis de la función tiroidea mostró hipertiroidismo, como es habitual en los casos de tiroiditis piógena, con ulterior normalización de los valores de TSH tras la resolución del cuadro. La aspiración con aguja fina (FNA) realizada en este caso, fue útil para obtener el cultivo del germen y dirigir la terapia médica. En cuanto al tratamiento combinado (médico y quirúrgico) se ha encontrado que del 64\% de los pacientes llevados a punción y drenaje, el 94\% requirió cirugía adicional, incluida la cirugía de cuello abierto para drenaje.

En conclusión, la tiroiditis supurativa es una patología muy infrecuente que suele aparecer sobre una tiroides de ecoestructura alterada, o con patología tiroidea de base, por ejemplo, fístulas del seno piriforme y, especialmente, en pacientes inmunodeprimidos. Nuestro caso no cumplía ninguna de estas características, lo que lo hace aún más inusual.

No se encontraron datos de la epidemiologia de esta patología en Colombia.

\section{Tiroiditis}

\section{Introducción}

El término tiroiditis (o inflamación de la glándula tiroides) engloba una amplia gama de enfermedades cuyo origen, manifestaciones y tratamiento difieren de forma notable. Las tiroiditis se clasifican según el tiempo de evolución (agudas, subagudas y crónicas), su presentación clínica (supurada, silente, posparto), su etiología (infecciosa, autoinmune o desconocida), o por otras características que incluyen desde nombres propios (Hashimoto, De Quervain, Riedel) hasta aspectos anatomopatológicos (granulomatosa, linfocítica, atrófica). Además, hay tiroiditis que no encajan en este esquema, como las tiroiditis de origen iatrogénico, ya sea por fármacos (entre los que destaca amiodarona, litio, interferón alfa o interleucina 2) o posradiación.

Desde el punto de vista funcional, las tiroiditis pueden asociarse con cualquier presentación. Las variadas formas de tiroiditis pueden causar tirotoxicosis, hipotiroidismo o no alterar la función tiroidea. Otro enfoque de clasificación aún más práctico es clasificarla en aquellas tiroiditis que puedan causar dolor o no.

A continuación, nos referiremos a las tiroiditis más comunes en la práctica clínica (tabla $\mathbf{1}$ ). 
Tabla 1. Tipos de tiroiditis

\begin{tabular}{|c|c|c|c|c|}
\hline Tipo & Causa & Presentación clínica & Diagnóstico & Duración y resolución \\
\hline Tiroiditis de Hashimoto & Anticuerpos antitiroideos & $\begin{array}{l}\text { Hipotiroidismo } \\
\text { Rara tirotoxicosis } \\
\text { transitoria }\end{array}$ & $\begin{array}{l}\text { Prueba de función } \\
\text { tiroidea, anticuerpos }\end{array}$ & $\begin{array}{l}\text { Hipotiroidismo } \\
\text { permanente }\end{array}$ \\
\hline $\begin{array}{l}\text { Tiroiditis subaguda } \\
\text { (Quervain) }\end{array}$ & Posible causa viral & $\begin{array}{l}\text { Tiroiditis dolorosa, } \\
\text { tirotoxicosis seguida de } \\
\text { hipotiroidismo }\end{array}$ & $\begin{array}{l}\text { Prueba de función } \\
\text { tiroidea, VSG, captación } \\
\text { de yodo }\end{array}$ & $\begin{array}{l}\text { Resolución en } 12-18 \text { meses } \\
\text { (eutiroidismo) } \\
5 \% \text { de posibilidad } \\
\text { de hipotiroidismo } \\
\text { permanente }\end{array}$ \\
\hline $\begin{array}{l}\text { Tiroiditis silente, tiroiditis } \\
\text { indolora }\end{array}$ & $\begin{array}{l}\text { Anticuerpos antitiroideos, } \\
\text { autoinmunidad }\end{array}$ & $\begin{array}{l}\text { Tirotoxicosis seguido de } \\
\text { hipotiroidismo }\end{array}$ & $\begin{array}{l}\text { Prueba de función } \\
\text { tiroidea, anticuerpos }\end{array}$ & $\begin{array}{l}\text { Resolución en } 12-18 \text { meses } \\
\text { (eutiroidismo) } \\
20 \% \text { de probabilidad } \\
\text { de hipotiroidismo } \\
\text { permanente }\end{array}$ \\
\hline $\begin{array}{l}\text { Inducida por } \\
\text { medicamentos }\end{array}$ & $\begin{array}{l}\text { Amiodarona, litio, interfe- } \\
\text { rón, ITK }\end{array}$ & $\begin{array}{l}\text { Tirotoxicosis o } \\
\text { hipotiroidismo }\end{array}$ & $\begin{array}{l}\text { Prueba de función } \\
\text { tiroidea, anticuerpos }\end{array}$ & $\begin{array}{l}\text { Continua durante la } \\
\text { administración del } \\
\text { medicamento }\end{array}$ \\
\hline $\begin{array}{l}\text { Tiroiditis aguda } \\
\text { Tiroiditis supurativa }\end{array}$ & $\begin{array}{l}\text { Principalmente bacteriana } \\
\text { Pueden ser por otros } \\
\text { gérmenes }\end{array}$ & $\begin{array}{l}\text { Tiroiditis dolorosa, sínto- } \\
\text { mas generales } \\
\text { Ocasionalmente } \\
\text { hipotiroidismo } \\
\text { Tirotoxicosis }\end{array}$ & $\begin{array}{l}\text { Prueba de función } \\
\text { tiroidea, ACAF } \\
\text { Captación de yodo }\end{array}$ & $\begin{array}{l}\text { Resolución al corregir la } \\
\text { causa infecciosa }\end{array}$ \\
\hline Tiroiditis posparto & Anticuerpos antitiroideos & $\begin{array}{l}\text { Tirotoxicosis seguida de } \\
\text { hipotiroidismo }\end{array}$ & $\begin{array}{l}\text { Prueba de función } \\
\text { tiroidea, anticuerpos } \\
\text { tiroideos } \\
\text { Captación de yodo } \\
\text { (contraindicado en } \\
\text { lactancia) }\end{array}$ & $\begin{array}{l}\text { Resolución de } 12 \text { - } \\
18 \text { meses. } 20 \% \text { de } \\
\text { probabilidad de } \\
\text { hipotiroidismo } \\
\text { permanente }\end{array}$ \\
\hline Inducida por radiación & $\begin{array}{l}\text { Postratamiento con yodo } \\
\text { radioactivo para hiperti- } \\
\text { roidismo } \\
\text { Tratamiento para cáncer }\end{array}$ & $\begin{array}{l}\text { Ocasionalmente } \\
\text { tirotoxicosis, más } \\
\text { frecuente hipotiroidismo }\end{array}$ & Prueba de función tiroidea & $\begin{array}{l}\text { Tirotoxicosis transitoria } \\
\text { El hipotiroidismo suele ser } \\
\text { permanente }\end{array}$ \\
\hline
\end{tabular}

ACAF: aspiración con aguja fina. Tomada de: Sweeney LB, Stewart C, Gaitonde DY. Thyroiditis: an integrated approach. Am Fam Physician. $2014 ; 90(6): 389-96$.

\section{Enfermedad tiroidea por IgG4}

Es una condición que afecta muchos órganos y sistemas, incluido el sistema endocrino, en general, y particularmente la glándula tiroides. Tiene predominio sobre el sexo masculino. Se divide en 4 subcategorías: tiroiditis de Riedel; variante fibrosante de tiroiditis de Hashimoto; tiroiditis de Hashimoto relacionada con IgG; y enfermedad de Graves con IgG4 elevada ${ }^{(7)}$.

Se caracteriza por un infiltrado linfoplasmocítico denso, con abundante IgG4, además de la formación de tumefacciones y fibrosis asociado con elevación de los niveles séricos de IgG4. Son múltiples las entidades asociadas con IgG4.

\section{Epidemiología}

La incidencia de la enfermedad es de 0,28\%-1,05\%/100 000 habitantes. En Japón se presentaron 8000 casos en 2009 con una relación hombre:mujer de 4:1. La edad más frecuente de aparición está entre los 50 y 70 años ${ }^{(7)}$.

\section{Fisiopatología}

Varios factores se han relacionado con esta entidad. Dentro de estos, los más importantes incluyen factores genéticos, respuestas mediadas por reacciones antígeno-anticuerpo y fenómenos alérgicos. En los factores genéticos se han descrito los relacionados con la pancreatitis autoinmune en la población japonesa y los asociados con la presencia de los haplotipos HLA-DRB1 * 0405 y HLA-DQB1 * 0401.

En la reacción antígeno anticuerpo y fenómeno alérgico se presenta una respuesta inicial inducida por un proceso de infección o por la presencia de alérgenos o microorganismos comensales, seguido por la infiltración del tejido por linfocitos T activos. Posteriormente, existe una producción de IL- 4, 
IL-5, IL-10, IL-13, IFN- $\gamma$, y TGF- $\beta$. Moléculas como IL4 e IL10 activan los linfocitos B para la producción de IgE e IgG4 IL-5, IL-13 y TGF- $\beta$ y realizan el reclutamiento de eosinófilos y la ulterior activación de fibroblastos. Luego, el IgE e IgG4 pueden reaccionar de forma cruzada con autoantígenos. Por último, los linfocitos B presentan autoantígenos a los linfocitos T autorreactivos (figura 3) ${ }^{(7)}$.

Figura 3. Mecanismo patológico de la enfermedad tiroidea por IgG
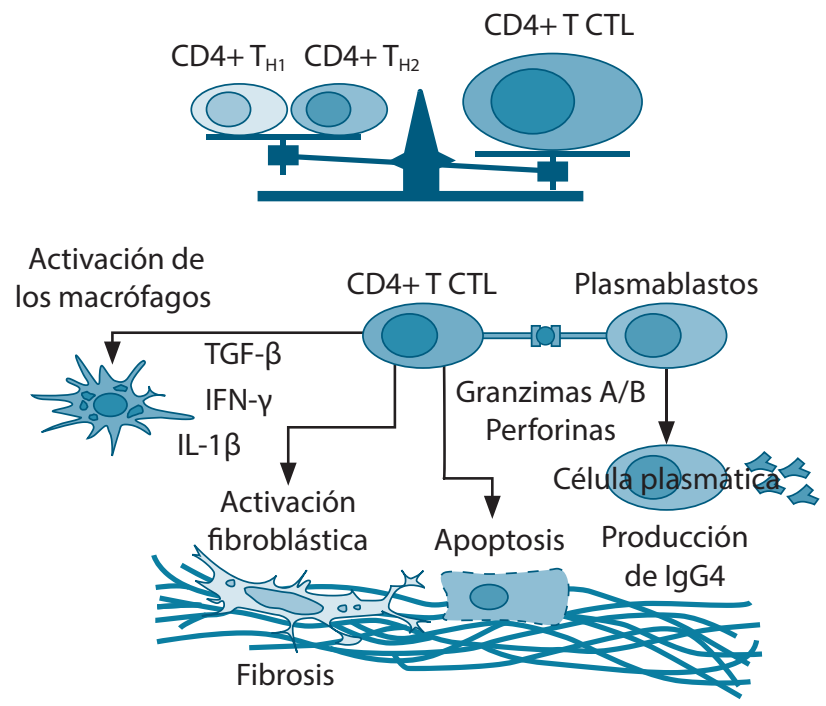

\section{Diagnóstico histopatológico y bioquímico}

Los criterios para el diagnóstico se presentan en la tabla 2.

Tabla 2. Diagnóstico histopatológico y bioquímico de la tiroiditis por IgG 4

Diagnóstico histopatológico Diagnóstico bioquímico

- Criterios mayores:

- Infiltrado linfoplasmocítico rico en $\lg \mathrm{G} 4$

- Niveles de lgG4 $>135 \mathrm{mg} / \mathrm{dL}$ (sensibilidad $90 \%$ y especificidad $60 \%)$

- Fibrosis dispuesta, al menos focalmente en patrón irregular

- Flebitis obliterante

- Criterios menores que pueden ser asociados, pero son inespecíficos:

- Flebitis sin obliteración del lumen

- Mayor número de eosinófilos

Para el diagnóstico seguro se necesitan 2 criterios mayores.

\section{Tiroiditis fibrosa o de Riedel}

Es una forma de tiroiditis muy infrecuente dada su incidencia de $0,06 \%$. Tiene mayor predilección por el sexo femenino en edades entre los 40-60 años. Se caracteriza por infiltración de la glándula tiroides, la cual se puede presentar como anormalidad aislada o como una manifestación de una enfermedad sistémica. Generalmente se presenta con un aumento progresivo de la tiroides, la cual es indolora, firme y bilateral y se puede confundir con otras alteraciones que afectan dicha glándula: tiroiditis autoinmune, bocio multinodular no tóxico, tiroiditis subaguda, tiroiditis infecciosa, cáncer de tiroides o linfoma. Como particularidad, esta entidad puede invadir los tejidos blandos peritiroideos ${ }^{(8)}$.

\section{Etiología y patogenia}

Esta tiroiditis está relacionada con la enfermedad sistémica por IgG4. Se desconoce su etiología, pero la afectación local de la tiroides puede ser secundaria a la presencia de enfermedad sistémica o asociada con enfermedad autoinmune. Se caracteriza por la presencia de un proceso fibrótico que incluye un infiltrado de células mononucleares, el cual se extiende más allá de la tiroides y compromete hasta el tejido blando peritiroideo; además, puede comprometer las glándulas paratiroides, nervio laríngeo recurrente, tráquea, mediastino y pared torácica anterior. En contraste, otras condiciones infiltrativas e inflamatorias de la tiroides no se extienden más allá de la cápsula tiroidea. Sin embargo, anticuerpos elevados pueden reaccionar a antígenos liberados por tejido tiroideo destruido ${ }^{(8)}$.

\section{Clínica}

Clínicamente la tiroiditis se presenta como una tumefacción de rápido crecimiento, de consistencia dura y que comprime estructuras vecinas. Produce dolor, disnea, disfagia y estridor laríngeo. El 64\% de los pacientes es eutiroideo, 32\% de los pacientes presenta hipotiroidismo y solo $4 \%$ hipertiroidismo. El 60\% puede presentar autoanticuerpos elevados. Además, puede ocurrir asociada con otras afecciones fibrosas: fibrosis retroperitoneal, mediastinitis fibrosa, colangitis esclerosante, pancreatitis, fibrosis lagrimal y pseudotumoral orbital y las lesiones fibroinflamatorias tumefactivas de la cabeza y cuello.

\section{Diagnóstico}

El diagnóstico clínico es difícil porque simula una neoplasia o la variante fibrosa de la tiroiditis Hashimoto. Puede producir hipoparatiroidismo por compromiso de las glándulas paratiroideas o síndrome de vena cava superior si su extensión es mayor. Desde el punto de vista imagenológico disponemos de varios estudios. La TAC permite valorar la extensión de la fibrosis y la compresión de la tráquea o el esófago. En el PET se evidencia aumento del metabólico glucídico. Es útil para valorar la actividad de la enfermedad y la respuesta a los corticoides. La gammagrafía tiroidea en esta entidad presenta 
captación heterogénea o muy baja. La ecografía tiroidea muestra lesiones focales o diseminadas, hipoecoicas con compromiso muscular y ausencia y flujo vascular en las áreas afectadas.

El diagnóstico se suele hacer con biopsia Tru-Cut, dado que la PAF es difícil por la consistencia del tejido ${ }^{(9)}$.

\section{Tratamiento}

El tratamiento está encaminado a corregir el hipotiroidismo y mejorar los síntomas relacionados con la fibroesclerosis. El tratamiento de la fibroesclerosis es empírico. Se han empleado varios esquemas de tratamiento:

- Primera línea: dosis de metilprednisolona 15-1000 mg/ diarios (la suspensión ocasiona recidiva)

- Segunda línea: utilización de tamoxifeno, el cual estimula la producción de TGFß e inhibe la proliferación fibroblástica y epitelial. Se utiliza en dosis de 10-40 mg.

- Tercera línea: rituximab, el cual puede ser utilizado en casos de fallo a la terapia anterior. La cirugía descompresiva se utiliza en casos de compresión o compromiso de la vía aérea ${ }^{(8,9)}$

\section{Tiroiditis subaguda granulomatosa}

También llamada por otros nombres: tiroiditis no supurativa subaguda, tiroiditis de Quervain o tiroiditis granulomatosa subaguda. Es una causa poco común de hipertiroidismo. Se encuentra relacionada en su presentación con la enfermedad de Graves de 1:5-10 casos y es más frecuente en mujeres, con una relación 7:1.

\section{Etiología}

Como antecedente encontramos una historia previa de infección de las vías aéreas superiores, la cual puede estar presente de 2-8 semanas antes y que precede a la tiroiditis. Se encuentra asociada con epidemias de virus Coxsackie y otras infecciones virales, además de la existencia de una fuerte asociación con el antígeno leucocitario humano (HLA) -B35. Los virus más frecuentemente asociados son parvovirus, Coxsackie, sarampión, citomegalovirus (CMV), adenovirus, echovirus. Otro mecanismo etiopatogénico es la teoría del mimetismo molecular o daño tisular por los virus que se unen al HLA B35 de los macrófagos, que activan los LT citotóxicos y producen daño en la célula folicular. Una característica importante es que la respuesta inmune no se perpetua, lo que hace que este proceso sea autolimitado ${ }^{(8,10)}$.

\section{Patología}

Macroscópicamente existe inflamación y aumento del tamaño de la glándula tiroidea. A nivel microscópico se presenta infiltración de neutrófilos, linfocitos, histiocitos y células gigantes, con ruptura y colapso folicular.

\section{Clínica}

El patrón clásico de esta entidad es trifásico: un período de hipertiroidismo inicial seguido de hipotiroidismo y ulterior recuperación. La sintomatología perdura mientras los niveles de T4 y T3 se agotan, aproximadamente entre 2-6 semanas (figura 4).

Figura 4. Patrón clásico de la tiroiditis

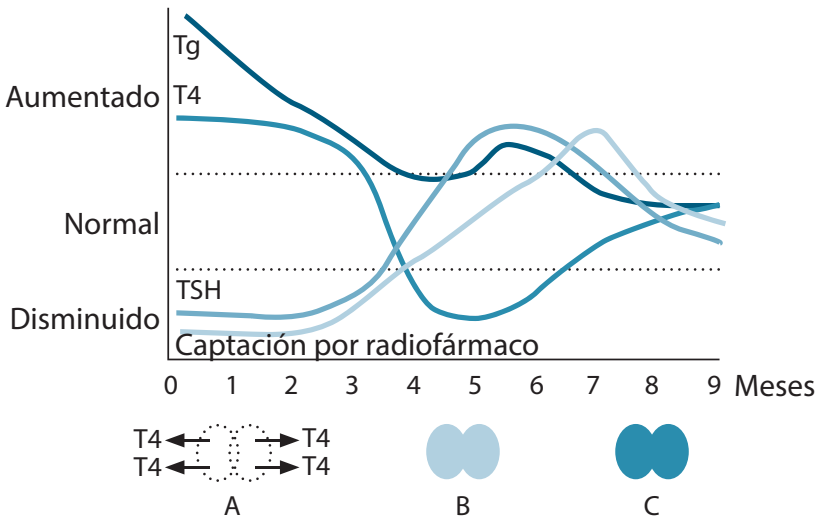

Tomada de: Galofré, JC, Pineda J, Toni M, et al. Tiroiditis. Medicine-Programa de Formación Médica Continuada Acreditado. 2016;12(13):742-53.

\section{Patrón trifásico en tiroiditis subaguda}

Liberación masiva de T4 y Tg, con elevación de sus niveles, lo que produce la fase de tirotoxicosis inicial. En esta fase, el exceso de T4 inhibe la secreción de TSH, lo que hace que los niveles estén suprimidos. Además, la glándula tiroidea, debido a la destrucción inflamatoria, pierde su capacidad para almacenar isótopos radiactivos, por lo cual existe ausencia de captación. Luego, se produce la degradación de T4 que se ha ido liberando $\mathrm{y}$ tras un fugaz estado de eutiroidismo, el paciente entra en la segunda fase (hipotiroidismo), ya que la T4 desciende. La hipófisis aumenta la producción de TSH con el objetivo de estimular la producción de T4 por parte de una tiroides insuficiente que no es capaz de sintetizar de nuevo T4. Tras varios meses, la tiroides recupera su capacidad para sintetizar hormonas y se restablece la función tiroidea hasta normalizar su función.

El comienzo de los síntomas es súbito, con presencia de dolor intenso local o con irradiación cervical, maxilar y auricular. Puede presentarse fiebre, anorexia, mialgias y mal estado general. El $50 \%$ al $60 \%$ de los pacientes presenta tirotoxicosis manifiesta. La inflamación puede afectar toda la tiroides o solo a un lóbulo y muy raramente puede evolucionar a una crisis tirotóxica.

\section{Diagnóstico}

El perfil bioquímico del hipertiroidismo se presenta en la fase inicial con T3 y T4L medianamente elevadas. Existe elevación transitoria de la VSG con niveles usualmente $>50 \mathrm{~mm} / \mathrm{h}$. Con frecuencia, la función hepática suele estar alterada en la 
fase inicial, relacionado con la infección viral, además de la presencia de leucocitosis y anemia moderada. Desde el punto de vista imagenológico, en la gammagrafía encontramos baja captación del radio yodo o una glándula tiroidea normal o aumentada de forma difusa y con bajo flujo durante la fase de hipertiroidismo ${ }^{(10)}$.

\section{Tratamiento}

El tratamiento está encaminado al manejo del dolor. Si se presentan síntomas hipertiroideos se pueden administrar medicamentos bloqueantes $\beta$ dado que los síntomas no serán muy intensos. En la fase hipotiroidea solo se da tratamiento si los niveles de TSH son mayores de 10 con síntomas de disfunción. Bajo esta condición se puede administrar levotiroxina en dosis de 50-100 $\mu$ g durante 2 a 8 semanas y revalorar la condición para descartar hipotiroidismo permanente.

El diagnóstico diferencial debe descartar tiroiditis aguda infecciosa, otras causas de hipertiroidismo hipocaptante, tiroiditis autoinmune (Hashimoto o Graves), carcinoma tiroideo o linfoma ${ }^{(8)}$.

\section{Tiroiditis posparto}

Se define como la ocurrencia de tirotoxicosis seguida de hipotiroidismo o la presencia de solo hipotiroidismo en una mujer hasta 1 año después del parto (se incluye postaborto). La prevalencia es de aproximadamente el 8\% en los embarazos. Las mujeres que presentan tiroiditis posparto tienen hasta el 30\% de riesgo de desarrollar hipotiroidismo permanente entre 5-10 años después del episodio. Además, en las mujeres con hipertiroidismo clínico se debe distinguir de las exacerbaciones posparto de la enfermedad de Graves.

\section{Patogénesis}

Se presenta infiltración linfocítica con características histológicas similares a las del Hashimoto, sin el mismo grado de fibrosis o atrofia folicular. Hay evidencia de autoinmunidad tiroidea antes y después de la gestación: presencia de anticuerpos antiperoxidasa tiroidea (TPO) (+) (inducen citotoxicidad mediada por reacción antígeno-anticuerpo). El 12\%-26\% de las mujeres podría tener anticuerpos TPO positivos. Si los presentan en el primer trimestre tendrán un riesgo de 33\% al $55 \%$ de desarrollar tiroiditis posparto. Estas pacientes tienen una relación CD4/CD8 más alta y mayor número de linfocitos T activados. Además, se maneja la teoría del microquimerismo fetal, que es la presencia de células fetales que poseen antígenos maternos y paternos, los cuales se depositan en la tiroides materna, desde donde desencadenan autoinmunidad en el posparto. También se presentan factores ambientales asociados con la aparición de tiroiditis posparto, como el consumo de tabaco, toxicidad por yodo y la deficiencia de selenio.

\section{Clínica}

La fase hipotiroidea se presenta entre 4-8 meses después del parto y dura entre 4 y 6 meses. Se puede presentar hipotiroidismo permanente en aproximadamente el 25\% de las mujeres. Los síntomas más frecuentes son fatiga, disfunción cognitiva (o depresión) e intolerancia al frío. Solo el hipertiroidismo con resolución espontánea puede ocurrir en 32\% (entre la primera y sexta semana posparto, duración hasta 2 meses) e hipotiroidismo con resolución espontánea en 43\% (entre el tercer y noveno mes posparto, con duración de hasta 4 meses). También puede presentarse hipertiroidismo seguido de hipotiroidismo en $25 \%-30 \%$ de las afectadas y permanecer en estado hipotiroideo durante 1 año posparto. Hay que diferenciar entre tiroiditis posparto hipertiroidea y la enfermedad de Graves ${ }^{(11)}$.

\section{Diagnóstico}

Dentro del diagnóstico bioquímico se puede encontrar niveles de T3 y T4 elevados o en el límite superior de la normalidad, con una TSH suprimida o, de forma contraria, según se encuentre: hiper o hipotiroidea. Anticuerpos antiTPO pueden estar presentes hasta en el $80 \%$ de los casos y suelen estar más elevados durante la fase de hipotiroidismo. Dentro de los métodos imagenológicos encontramos la gammagrafía tiroidea, en la cual está baja la radiocaptación en la fase hipertiroidea (contrario al Graves). No suele ser necesario realizarlo dado que la fase hipertiroidea se suele diagnosticar en retrospectiva $^{(11)}$.

\section{Tratamiento}

La mayoría de las pacientes no requiere tratamiento, pero si presenta hipertiroidismo muy sintomático, las pacientes serían candidatas a recibir tratamiento con bloqueantes $\beta$. El propanolol es el medicamento de elección durante la lactancia. Para pacientes con hipotiroidismo sintomático o TSH $>10$ iniciar manejo con levotiroxina en dosis de 50-100 $\mu \mathrm{g}$ /día.

Las mujeres con TSH menor de 10, asintomáticas y que no estén planeando un siguiente embarazo no requieren intervención. Se debe monitorizar la función tiroidea cada 4-8 semanas para determinar la resolución bioquímica o si se presenta hipotiroidismo permanente. La duración del tratamiento debe recomendarse hasta 6 semanas después de iniciado, pero dependerá del seguimiento individual de cada paciente ${ }^{(8)}$.

\section{Tiroiditis infecciosa}

Las tiroiditis infecciosas son infrecuentes y afectan principalmente a pacientes con estado de inmunosupresión. El 85\% de estas es bacteriana, $15 \%$ micótica y $5 \%$ parasitaria. Por lo general se presentan por diseminación hematógena a través de una fístula en el seno piriforme o del seno del cuarto arco 
branquial o por la presencia de una fístula en el conducto tirogloso persistente. También se puede desarrollar luego de procedimientos cervicales, colocación de catéter venoso central o biopsia por aspiración con aguja fina.

\section{Etiología}

Anatómicamente existen varias vías de diseminación:

- Fístula del seno piriforme

- Diseminación linfática o hematógena (incluida la embolia séptica), aumento del riesgo por alteraciones anatómicas de la tiroides (nódulo tiroideo o cáncer)

- Extensión directa de un absceso

- Trauma cervical

- Inoculación directa de la tiroides o la anatomía próxima (uso de medicamentos IV, estudios intervencionistas, como ACAF o colocación de CVC)

- Infección secundaria

- Ruptura esofágica

- Ingestión de cuerpo extraño con perforación esofágica

- Absceso retrofaríngeo

- Alteraciones anatómicas de la glándula tiroides.

\section{Diagnóstico}

Los estudios imagenológicos pueden ser utilizados como apoyo. La TAC permite valorar la extensión del compromiso y las estructuras vecinas y es la primera elección en muchos centros. La ecografía de tiroides es de elección en pacientes sin compromiso de la vía aérea. Permite el diagnóstico microbiológico y tratamiento mediante ACAF. La aspiración con aguja de la colección se utiliza para drenaje y obtener los cultivos de la misma. Además, se realizará fistulectomía en los casos que sea necesario. Se debe evitar el uso de corticoides, ya que pueden agravar el proceso ${ }^{(12)}$.

\section{Tratamiento}

En caso de persistencia de síntomas dado por deterioro clínico y dos ecografías con FNA sin mejoría clínica, la indicación de drenaje abierto, dado el riesgo de perforación esofágica, extensión a mediastinitis necrotizante o sepsis, es la mejor opción. La tiroidectomía parcial o total solo debe ser considerada en casos de abscesos múltiples, pobremente definidos y con persistencia de deterioro del estado general; tejido tiroideo necrótico o sospecha de malignidad subyacente ${ }^{(12)}$.
Una sugerencia para la recomendación de algoritmo incluye:

- Primera etapa: diagnóstico diferencial, monitorización de la vía aérea y cardíaco, paraclínicos y estudio de TAC contrastado.

- Segunda etapa: drenaje transcutáneo o abierto en pacientes con compresión de la vía aérea. Se debe considerar manejo conservador con antibióticos según la condición del paciente.

- Tercera etapa: manejo final. Drenaje quirúrgico en casos de persistencia. Tiroidectomía solo en casos de enfermedad muy extensa y corrección de fístulas de seno piriforme, si es el caso.

La evaluación y manejo de la tiroiditis aguda se presentan en las figuras 5, 6, 7 y 8.

Figura 5. Evaluación aguda y manejo de la tiroiditis aguda supurativa ${ }^{(12)}$

\section{Evaluación aguda y manejo de la tiroiditis} aguda supurativa bacteriana

\section{Sospecha de tiroiditis supurativa aguda}

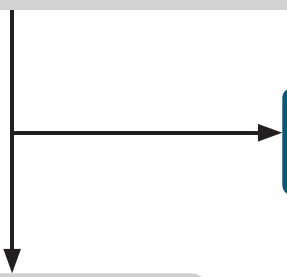

Monitorizar vía área/

cardiovascular

$\mathrm{CH}$, electrólitos, BUN,

Cr, función hepática,

hemocultivos, serología

VIH, VSG, PCR

Considerar diagnósticos

diferenciales

Inicio de antibioticoterapia

empírica

Estudio de TAC de cuello y tórax contrastado

CV

Considerar

laringoscopia por fibra óptica transnasal

RM

Trago de bario

Gl alta

Imágenes específicas basadas en la sospecha de posible fuente de infección

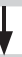

Sospecha de tiroiditis supurativa aguda 
Figura 6. Evaluación secundaria y manejo de tiroiditis aguda supurativa ${ }^{(12)}$

\section{Evaluación secundaria y manejo de la tiroiditis aguda supurativa bacteriana}

Valoración de la vía aérea del paciente

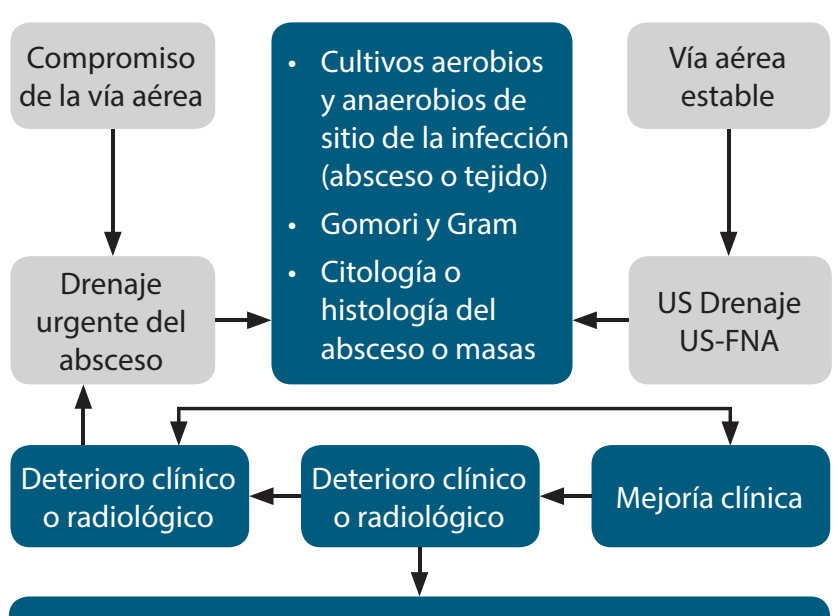

Antibioticoterapia específica

Continuar con el manejo a largo plazo
Figura 7. Manejo a largo plazo de la tiroiditis aguda ${ }^{(12)}$

\section{Manejo a largo plazo de tiroiditis aguda supurativa} bacteriana

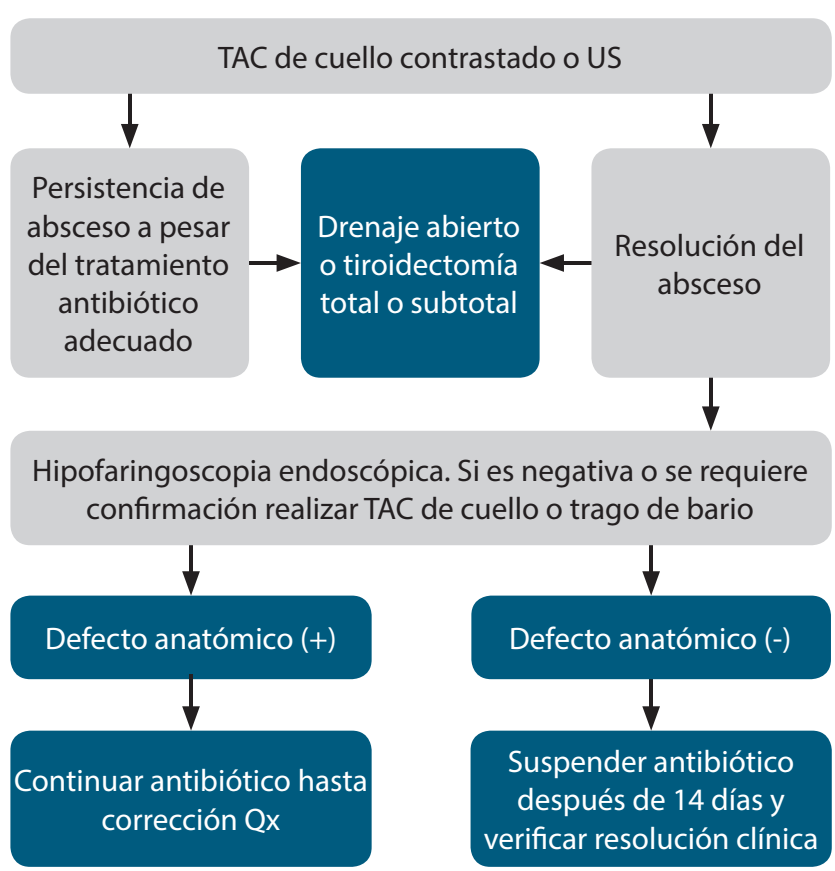

Figura 8. Enfoque de la tiroiditis

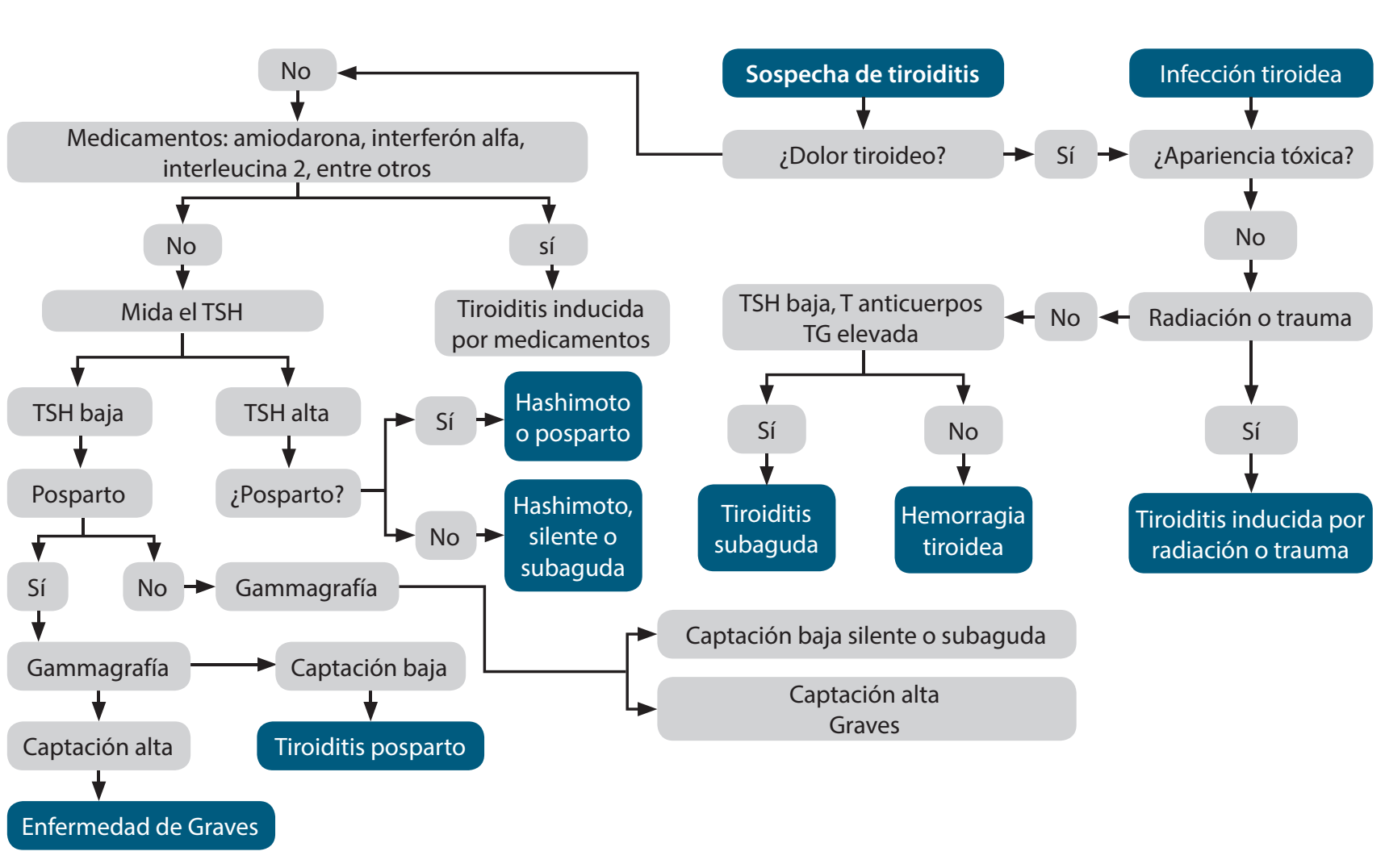




\section{Responsabilidades éticas}

\section{Confidencialidad de los datos}

Los autores declaran que han seguido los protocolos de su centro de trabajo sobre la publicación de datos de la paciente y que esta recibió información suficiente y ha dado su consentimiento informado para participar en dicho reporte.

\section{Conflicto de intereses}

Los autores declaran no tener ningún conflicto de intereses.

\section{Referencias}

1. Cawich SO, Hassranah D, Naraynsingh V. Idiopathic thyroid abscess. Int J Surg Case Rep. 2014;5(8):484-6.

2. Gady J, Piorkowski R. Infections of the thyroid gland. En: Hupp J, Ferneini EM (editor). Head, neck, and orofacial infections. 2016. p. 288-92.

3. Bravo E, Grayev A. Thyroid abscess as a complication of bacterial throat infection. J Radiol Case Rep. 2011;5:1-7.

4. Schweitzer VG, Olson NR. Thyroid abscess: otolaryngol. Head Neck Surg. 1981;89(2):226-9.

5. Jonas NE, Fagan JJ. Internal jugular vein thrombosis: a case study and review of the literature. Internet J Otorhinolaryngol. 2007;6:2.

6. Yedla N, Pirela D, Manzano A, Tuda C, Lo Presti S. Thyroid abscess: challenges in diagnosis and management. J Investig Med High Impact Case Rep. 2018;6.

7. Kottahachchi D, Topliss DJ. Immunoglobulin G4-related thyroid diseases. Eur Thyroid J. 2016;5(4):231-9.

8. Oroog A, Truran P, Aspinall S. Thyrotoxicosis and thyroiditis. Surgery (Oxford). 2017;35(10):569-75.

9. Falhammar H, Juhlin CC, Barner C, Catrina SB, Karefylakis C, Calissendorff J. Riedel's thyroiditis: clinical presentation, treatment and outcomes Henrik Falhammar. Endocrine. 2018;60(1):185-92.

10. Rizzo L, Mana DL, Bruno OD. Tiroiditis no-autoinmunes. Medicina. 2014;74(6):481.

11. Keely EJ. Postpartum thyroiditis: an autoimmune thyroid disorder which predicts future thyroid health. Obstet Med. 2011;4(1):7-11.

12. Miyauchi A. Thyroid gland: a new management algorithm for acute suppurative thyroiditis? Nat Rev Endocrinol. 2010;6(8):424-6.

\section{Lecturas recomendadas}

- $\quad$ Elorza JL, Echenique-Elizonda M. Acute suppurative thyroiditis. J Am Coll Surg. 2002;195:729-30

- García JC, Redondo J, Civera M, Verdú J, Pellicer V, Martínez MP. Management of thyroid gland abscess. Acta Otorrinolaringol. 2018;70(2):61-7.

- Goldani LZ, Zavascki AP, Maia AL. Fungal thyroiditis: an overview. Mycopathologia. 2006;161:129-39.

- Jeng LB, Lin JD, Chen MF. Acute suppurative thyroiditis: a ten year review in a Taiwanese hospital. Scand J Infect Dis. 1994;26:297-300.

- Mali VP, Prabhakaran K. Recurrent acute thyroid swellings because of pyriform sinus fistula. J Pediatr Surg. 2008;43:e27-30.

- $\quad$ McAninch EA, Xu C, Lagari VS, Kim BW. Coccidiomycosis thyroiditis in an immunocompromised host post-transplant: case report and literature review. J Clin Endocrinol Metab. 2014;99:1537-42.

- $\quad$ Paes JE, Burman KD, Cohen J, Franklyn J, McHenry CR, Shoham S, et al. Acute bacterial suppurative thyroiditis: a clinical review and expert opinion. Thyroid. 2010;20(3):247-55.

- Pearce EN, Farwell AP, Braverman LE. Thyroiditis. N Engl J Med. 2003;348(26):2646-55.

- $\quad$ Seo JH, Park YH, Yang SW, Kim HY. Refractory acute suppurative thyroiditis secondary to pyriform sinus fistula. Ann Pediatr Endocrinol Metab. 2014;9:104-7.

- $\quad$ Sheu SY, Schmid KW. Inflammatory diseases of the thyroid gland. Epidemiology, symptoms and morphology. Pathologe. 2003;24(5):339-47.

- Sioka E, Efthimiou M, Skoulakis C, Zacharoulis D. Thyroid abscess requiring emergency intervention. J Emerg Med. 2011;43(6):e455-6.

- $\quad$ Suprabha J, Vijay K, Shital P. Acute bacterial thyroid abscess as a complication of septicemia. IDCP. 2000;9:383-6. 